\title{
Advanced nano- and biomaterials in biophysical chemistry
}

\author{
Libuše Trnková $^{1} \cdot$ Zdeněk Farka $^{2}$
}

Received: 20 September 2017/ Accepted: 20 September 2017/Published online: 11 October 2017

(C) Springer-Verlag GmbH Austria 2017

This Special Issue of Chemical Monthly comprises selected peer-reviewed and revised papers, presented at the 16th Workshop of Physical Chemists and Electrochemists (WOPCE) held in Brno, Czech Republic in May 2016. The articles contribute to the current interest in nano- and bioresearch and all associated with biophysical chemistry, combining aspects of chemistry, biology, physics, medicine, and ecotoxicology. It should be kept in mind that understanding the function and application of novel materials in biosciences would not be possible without advanced biophysical approaches. Nanoobjects are highly sensitive to changes in surface properties due to the high surface area and fast electron transfer supporting the biophotoelectrocatalysis. For this field, situated at the boundary between bioelectrochemistry and photoelectrochemistry, it is crucial that the nanoobjects' electrical and optical properties are tunable by manipulating their size and charge. The sophisticated interconnection of biomaterials such as nucleic acids, proteins, and membranes with nanomaterials (quantum dots, carbon-based, metal, and metal-oxide nanoobjects) allows the development of a new generation of devices with immense application potential. Nanoparticles can be expected to contribute towards photoactive bioelectrocatalytic cascades in specially controlled biorecognition systems, resulting in special smart sensors. However, in diagnostics, therapeutics, and theranostics, one must assume

Libuše Trnková

libuse@chemi.muni.cz

1 Department of Chemistry, Masaryk University, Kamenice 5, 62500 Brno, Czech Republic

2 CEITEC MU, Masaryk University, Kamenice 5, 62500 Brno, Czech Republic the possible harmful effects of nanomaterials on human health and safety. In this context, relevant studies should consider the biophysical responses of biomolecules, because their structures can be changed by interactions with the given nanomaterial.

A number of papers in this special issue address the possibility to utilize the diversity and small size of nanoparticles in various fields. Specific optical and electronic features enable nanomaterials to be employed in biolabeling, biological assays, and imaging; for example, quantum dots (QDs), which exhibit such properties, can be covalently conjugated with antibodies, enzymes, DNA, or peptides. The conjugates of CdTe QDs with bovine serum albumin may reduce the luminescence of QDs and the quenching effect can be observed. Silver nanoparticles facilitate the microbial disinfection and new technological concepts are suggested for this purpose. To degrade the opportunistic pathogen of the urogenical tract (Candida glabrata) nanostructured $\mathrm{TiO}_{2}$ decorated with silver quantum dots was used. The nanocomposite of $\mathrm{WO}_{3-\mathrm{x}}$ nanoneedles and $\mathrm{Fe}_{2} \mathrm{O}_{3}$ nanoparticles is utlized for the detection of volatile organic compounds, such as methanol, ethanol, acetone, and toluene. Fluorescence detection, implemented into a chromatographic system allows to analyze liquid or gaseous analytes. Graphene-based materials attract major attention due to their unique properties capable of improving (bio)sensors' characteristics, including the sensitivity, selectivity, and stability.

The europium ternary complex $\left[\mathrm{Eu}(\mathrm{DO} 3 \mathrm{~A})\left(\mathrm{CO}_{3}\right)\right]^{2-}$ can serve as an indirect carbonate sensor for clinical samples using a sensitive luminescence technique. Based on this stable complex, the determination of both urea and urease is made possible. Electroactive iminoboronates containing ferrocene were synthesized. Based on their 
electrochemical properties, different regioisomers were shown to exhibit different behavior due to the interaction of the boronic acid moiety with the adjacent imine group of the iminoferrocenyl residue.

The connecting link of several biomedical-oriented papers is cytochrome $\mathrm{P} 450$, the enzyme having many important roles in biochemical processes, e.g., (a) the detoxification and activation of the human carcinogen benzo $[a]$ pyrene; (b) the activation of the anticancer agent ellipticine resulting in DNA adduct formation; and (c) the oxidation as well as reduction of the herbal drugs aristolochic acid I and II. The other biomedical papers have also interesting topics. Dihydromyricetin is a flavonoid which is gaining popularity as an alcohol-fighting drug. However, it may interact with enzymes involved in the metabolisms of xenobiotics. An appropriate animal model is required to develop an antibody against bacterial lung infections for the treatment of cystic fibrosis (CF) patients.
The intratracheal instillation of enzyme neuraminidase in mice can increase the lung susceptibility towards Pseudomonas aeruginosa infection that mimics the conditions of CF patients.

On the molecular and cellular levels, the DNA interactions with regulatory proteins are highly important. To better understand the DNA-protein interactions, including the recombination, replication, transcription, and repair of damaged DNA, many attempts have been carried out to characterize the binding kinetics and affinity. Atomic force spectroscopy and surface plasmon resonance techniques are demonstrated to be capable of visualizing individual biomacromolecules and for kinetic analysis of their reactions.

We believe that the papers published in this special issue of Chemical Monthly will attract scientists in the field of biophysical and material chemistry and provide them stimulating ideas for their further scientific work. 